\title{
Organization of Octopus Arm Movements: A Model System for Studying the Control of Flexible Arms
}

\author{
Yoram Gutfreund, ${ }^{1}$ Tamar Flash, ${ }^{2}$ Yosef Yarom, ${ }^{1}$ Graziano Fiorito, ${ }^{3}$ Idan Segev, ${ }^{1}$ and Binyamin Hochner \\ ${ }^{1}$ Department of Neurobiology and Center for Neuronal Computation, Institute of Life Sciences, Hebrew University, \\ Jerusalem 91904, Israel, '2Department of Applied Mathematics, The Weizmann Institute of Science, Rehovot 76100, \\ Israel, and 3Laboratorio di Neurobiologia, Stazione Zoologica "A. Dohrn" di Napoli, Napoli 80121, Italy
}

Octopus arm movements provide an extreme example of controlled movements of a flexible arm with virtually unlimited degrees of freedom. This study aims to identify general principles in the organization of these movements. Video records of the movements of Octopus vulgaris performing the task of reaching toward a target were studied. The octopus extends its arm toward the target by a wave-like propagation of a bend that travels from the base of the arm toward the tip. Similar bend propagation is seen in other octopus arm movements, such as locomotion and searching. The kinematics (position and velocity) of the midpoint of the bend in three-dimensional space were extracted using the direct linear transformation algorithm. This showed that the bend tends to move within a single linear plane in a simple, slightly curved path connecting the center of the animal's body with the target location. Approximately $70 \%$ of the reaching movements demonstrated a stereotyped tangential velocity profile. An invariant profile was observed when movements were normalized for velocity and distance. Two arms, extended together in the same behavioral context, demonstrated identical velocity profiles. The stereotyped features of the movements were also observed in spontaneous arm extensions (not toward an external target). The simple and stereotypic appearance of the bend trajectory suggests that the position of the bend in space and time is the controlled variable. We propose that this strategy reduces the immense redundancy of the octopus arm movements and hence simplifies motor control.

Key words: movement control; muscular-hydrostat; kinematics; octopus; cephalopods; motor program; flexible arm; arm extension; reaching movements
The control and coordination of arm movements in threedimensional (3D) space is a complicated task, a challenge to both biological and artificial systems. Increasing the number of degrees of freedom of the arm makes this task more complicated (Hollerbach, 1990a), and yet most biological movements involve a large number of degrees of freedom (in a rigid arm this number is directly related to the number and type of joints). An extreme case with especially high degrees of freedom is given by systems in which muscles are unattached to any internal or external skeleton whatsoever. Consequently, these structures are much more flexible than jointed limbs and have virtually unlimited degrees of freedom. Examples of such structures are cephalopod tentacles, some of the vertebrate tongues, and the elephant trunk. These structures are composed solely of muscles used to generate movement and provide the necessary skeletal support (Kier, 1982; Smith and Kier, 1989; Chiel et al., 1992). Kier and Smith (1985) have termed these structures muscular-hydrostats because they are composed of incompressible muscle tissue. They suggested that the production of movement and force in muscularhydrostats is dictated by the constraint that the volume remains constant.

The octopus arm is of special interest as a muscular-hydrostat,

\footnotetext{
Received May 30, 1996; revised Aug. 13, 1996; accepted Aug. 26, 1996.

This work was supported by the Office of Naval Research (N00014-94-1-0480) and by the Israel Academy of Sciences and Humanities (190/95-1). We thank Dr. A. Sigalov and the Visualization Center of the Hebrew University for their assistance in analyzing video images. We also thank Hanoch Meiri and Shira Oren for technical assistance.

Correspondence should be addressed to Yoram Gutfreund, Department of Neurobiology, Institute of Life Sciences, Hebrew University, Jerusalem 91904, Israel.
}

Copyright (C) 1996 Society for Neuroscience $0270-6474 / 96 / 167297-11 \$ 05.00 / 0$ because it combines extreme flexibility (an octopus arm can bend at any point and in any direction, and it can elongate, shorten, and twist) with a capability for executing various sophisticated motor tasks, such as building a shelter, manipulating small objects (Wells and Wells, 1957), and opening a jar (Fiorito et al., 1990). These combined capabilities of flexibility and precision are of interest to both physiologists and robotics engineers. As a first step toward understanding the principles of the motor control of the octopus arm, we have studied the kinematics of arm extension, which as we demonstrated is a stereotyped, simple, and reproducible movement.

One strategy for gaining insights into the principles of planning and control of a motor system is to look for kinematic invariance in movement trajectories (Atkeson and Hollerbach, 1985). This strategy, successfully applied to the study of multijoint human arm movements (Morasso, 1981; Flash and Hogan, 1985) and speech movements (Smith et al., 1995), has been used here to study octopus arm trajectories. The aim of our study is to identify common kinematic features, or stereotyped patterns, which characterize arm movements. We report here on such features in arm extension, which we show is executed by a forward propagation of a bend along the arm. This bend propagation is a basic movement pattern that is used in different behaviors, such as locomotion, searching, and reaching. The bend travels along a simple planar path connecting the body of the animal with the location of an external target. The velocity of this bend usually follows a stereotyped profile, which is appropriately scaled for different speeds and distances. The simple and stereotyped nature of the reaching movement can reduce the complexity of the control of the flexible arms of the octopus. 


\section{MATERIALS AND METHODS}

Experimental animals. Specimens of Octopus vulgaris were either caught on the Mediterranean shore by local fishermen or supplied by the Stazione Zoologica in Naples, Italy. The animals were maintained in $50 \times$ $50 \times 40 \mathrm{~cm}$ glass tanks containing artificial seawater. The water was circulated continuously in a closed system and filtered through coral dust and active charcoal. Water temperature was held at $16^{\circ} \mathrm{C}$ in a $12 \mathrm{hr}$ light/dark cycle. For this study we used three animals weighing 300, 470, and $700 \mathrm{gm}$. Before video recording, the animals were placed in a bigger glass tank $(80 \times 80 \times 60 \mathrm{~cm})$ with a water temperature of $18^{\circ} \mathrm{C}$. Video recording began only after the animals were well acclimatized to the new tank (after a few days).

Behavioral task and video recording. The trial started when a target, a green plastic disk of $2 \mathrm{~cm}$ diameter (see Fig. $1 \mathrm{~A}$, circle), was lowered into the water. The target was moved slightly to draw the attention of the octopus to it. The octopus either extended one or more arms or swam toward the target. Every few trials, the animal was rewarded with a piece of crab meat tied to the target.

Two video cameras were used to record the arm movements. The cameras viewed the subject from the same aquarium face with an angle of $\sim 90^{\circ}$ between them. Shutter speed of both cameras was set to $1 / 250$. The PAL superVHS video system was used, allowing a temporal resolution of $20 \mathrm{msec}$ between adjacent images. The video images from both cameras were combined into one image through a video mixer.

Those trials in which the octopus extended its arm toward the target were termed "successful trials," regardless of whether the arm hit or missed the target. Successive video images of successful trials were digitized and displayed on a Silicon Graphics work station. Points of interest were marked manually with the mouse cursor on each image. The coordinates of the points from both cameras were saved.

Transformation between camera coordinates and external XYZ coordinates. The positions of designated points in 3D space were obtained by applying the direct linear transformation (DLT) method, a method commonly used for obtaining 3D coordinates from two cameras. Briefly, each camera is characterized by 11 parameters. The relation between these parameters and the external $X Y Z$ coordinates is defined by the DLT equations as follows:

$$
\begin{aligned}
& x_{1}=\frac{P_{1} X+P_{2} Y+P_{3} Z+P_{4}}{P_{9} X+P_{10} Y+P_{11} Z+1}, \\
& y_{1}=\frac{P_{5} X+P_{6} Y+P_{7} Z+P_{8}}{P_{9} X+P_{10} Y+P_{11} Z+1},
\end{aligned}
$$

where $x_{1}$ and $y_{1}$ are the image coordinates of a designated point in the image of camera 1. $X, Y$, and $Z$ are the unknown 3D coordinates of that point, and $P_{1}-P_{11}$ are 11 camera parameters that are related to the camera position, orientation, and optical properties (Wood and Marshall, 1986; Woltring and Huiskes, 1990). In the beginning of each experiment, we recorded images of a $40 \times 20 \times 20 \mathrm{~cm}$ calibration cube made of aluminum rods. Fourteen light-emitting diodes were placed on the corners and midpoints of the cube and served as control landmarks. This structure was then removed, and the octopus movements were recorded approximately within the volume defined by the calibration cube and using the same camera setup. The image coordinates of at least six of the control landmarks were later used to calculate the $11\left(P_{1}-P_{11}\right)$ camera parameters. Cognizance of these parameters for both cameras enables one to solve the DLT equations and obtain the 3D coordinates of any point recorded by both cameras. The above transformation was repeated for sequential video images (with a $20 \mathrm{msec}$ interval), resulting in a description of the position of the designated points in both space and time. Note that the coordinates used to represent the movements are defined by a laboratory fixed-coordinate system, which is attached to the calibration cube and not to the animal or the aquarium.

Although the DLT transformation assumes ideal cameras, video cameras are expected to have some image distortions. It has been shown empirically that as long as the object location is limited to within the calibration volume, sufficient accuracy is achieved (Wood and Marshall, 1986). The accuracy of our set-up has been assessed empirically in both space and time. Spatial errors were estimated in each experiment. This was accomplished either by comparing the calculated positions of six known landmarks to their actual positions, or in other cases, by reconstructing the length of six rods, $20 \mathrm{~cm}$ long, placed within the same workspace. Data were collected only from trials in which the maximal spatial error or the difference between the measured and the actual length was $<1 \mathrm{~cm}$. Velocity errors were estimated by measuring the velocities of points moving at known speeds (see "Tangential velocity").

Determination of the plane of motion. A multiple linear regression method was used to determine the plane of motion. We calculated the coefficients $a_{0}, a_{1}$, and $a_{2}$ that give the best fit, in a least-square approximation, of the motion trajectory to a linear plane equation:

$$
Y=a_{0}+a_{1} X_{1}+a_{2} X_{2},
$$

where the $Y$ values are the coordinates of the data points along one of the axes for the dependent variable, and $X_{1}$ and $X_{2}$ values are the corresponding coordinates of the independent variables. For each movement we repeated the multiple regression analysis three times, each time using a different axis as a dependent variable $(Y)$. The plane of motion was determined by the coefficients $a_{0}, a_{1}$, and $a_{2}$, which gave the least-square error. The coefficient of determination $R^{2}$ was used to describe the fit of the data to a plane. An F-test was used to examine the statistical significance of $R^{2}$.

Finally, to present the data in a clear manner, the data coordinates were transformed to another coordinate system in which the origin is located at the first data point and one of the axes is perpendicular to the plane of motion.

Tangential velocity. The tangential velocity of a point, that is, its velocity in the direction of movement, was calculated from the position data. We first smoothed the data by fitting a fifth order polynomial to the projection of the points on the $X, Y$, and $Z$ axes as a function of time [one polynomial for $X(t)$, one for $Y(t)$, and one for $Z(t)]$. The six coefficients of these three polynomials were obtained by calculating the least-square equation, using the singular value decomposition algorithm (Press et al., 1992). Then, the tangential velocity $V_{\tan }$ was calculated from the derivatives of the smoothed $X(t), Y(t)$, and $Z(t)$ coordinates according to:

$$
V_{\tan }=\sqrt{\left(\frac{d X}{d t}\right)^{2}+\left(\frac{d Y}{d t}\right)^{2}+\left(\frac{d Z}{d t}\right)^{2}} .
$$

The accuracy of the tangential velocity calculations was estimated by measuring the velocity of a point moving at a known speed (an oscilloscope beam). It was found that within the speed range of $5-50 \mathrm{~cm} / \mathrm{sec}$ (the range of velocities measured in the experiment), the error increased slightly with velocity. The root mean square (RMS) of the error measured at a speed of $50 \mathrm{~cm} / \mathrm{sec}$ was $\pm 4 \%$, with a maximal error of $9 \%$.

\section{RESULTS}

Arm extension in the octopus is a fundamental component in various behaviors, such as locomotion, searching, and reaching toward a target. In all cases, the arm is extended in what seems to be a stereotyped and robust pattern. An example of an arm extended toward a target is shown in Figure $1 A$ (the target is marked by a circle). The four images, taken during the course of the movement, show that the arm is extended by using a wave-like propagation of a bend in the arm (arrow), which travels from the base of the arm toward its tip. The bend is always curved dorsally so that the sucking rings, located on the ventral side of the arm (arrowhead in Fig. 1A, frame 1), point in the direction of movement. In cases where the arm is already extended, as in Figure $1 B$, the movement is initiated by first creating a bend, which is formed by twisting the arm (arrow in Fig. 1B, frame 2), and then pulling the distal part backward. After this maneuver, the bend travels toward the tip of the arm in the desired direction, as demonstrated in Figure $1 A$.

We outline the extension of the arm by registering the movement of two designated points: (1) one of the eyes (marked in Fig. $1 A$ ) and (2) the midpoint of the bend, which we termed "bend-point" (marked in Fig. $1 A$ ). Figure $2 A$ depicts the path of these two points during a reaching task, at $20 \mathrm{msec}$ intervals. The octopus reached toward the target (marked by $X$ ) by moving the bend from the vicinity of its body outward. At the same time the octopus moved toward the target. This body movement is represented on the graph by the changes in eye location, which because the head of the octopus 
A
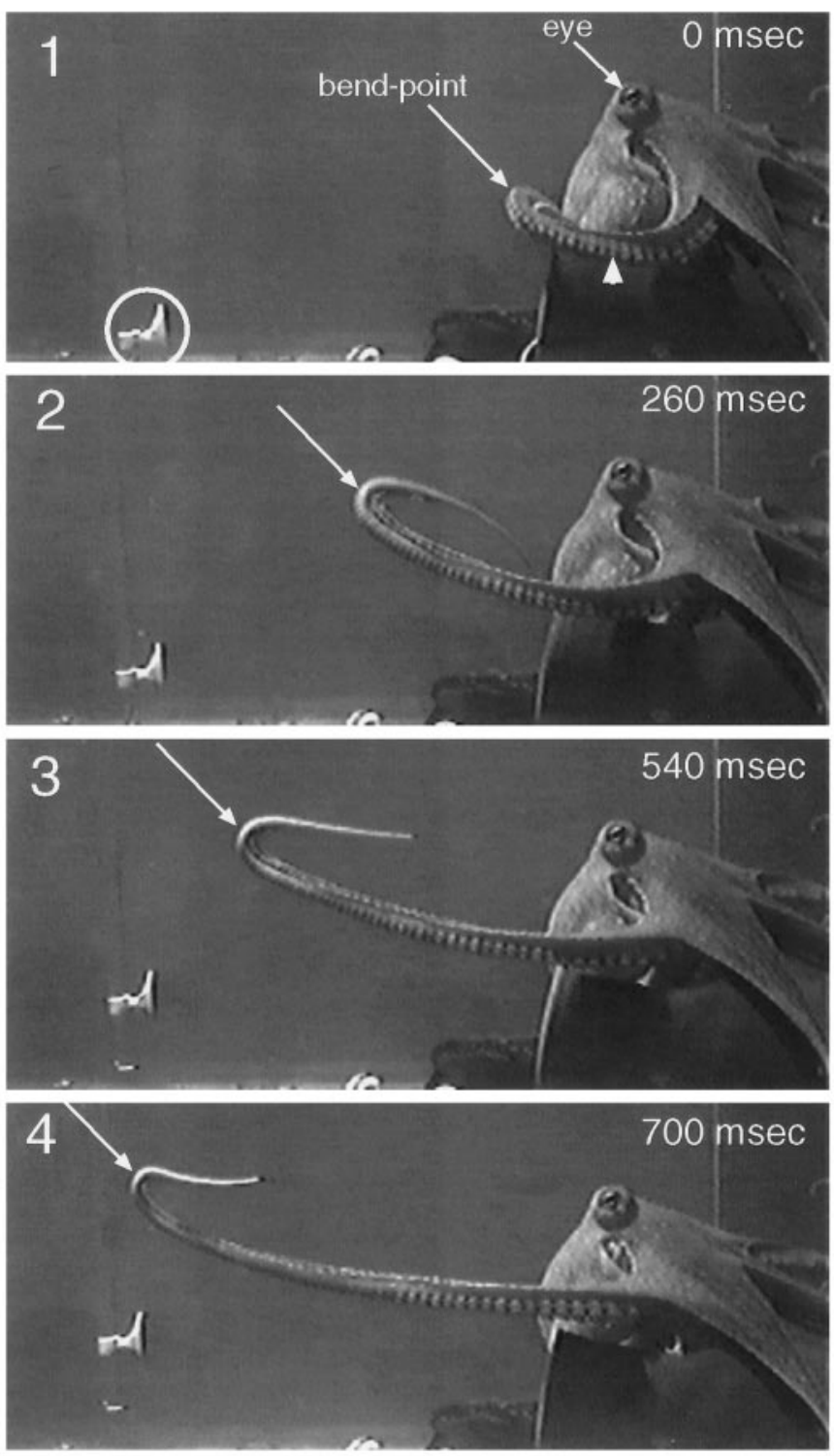

B
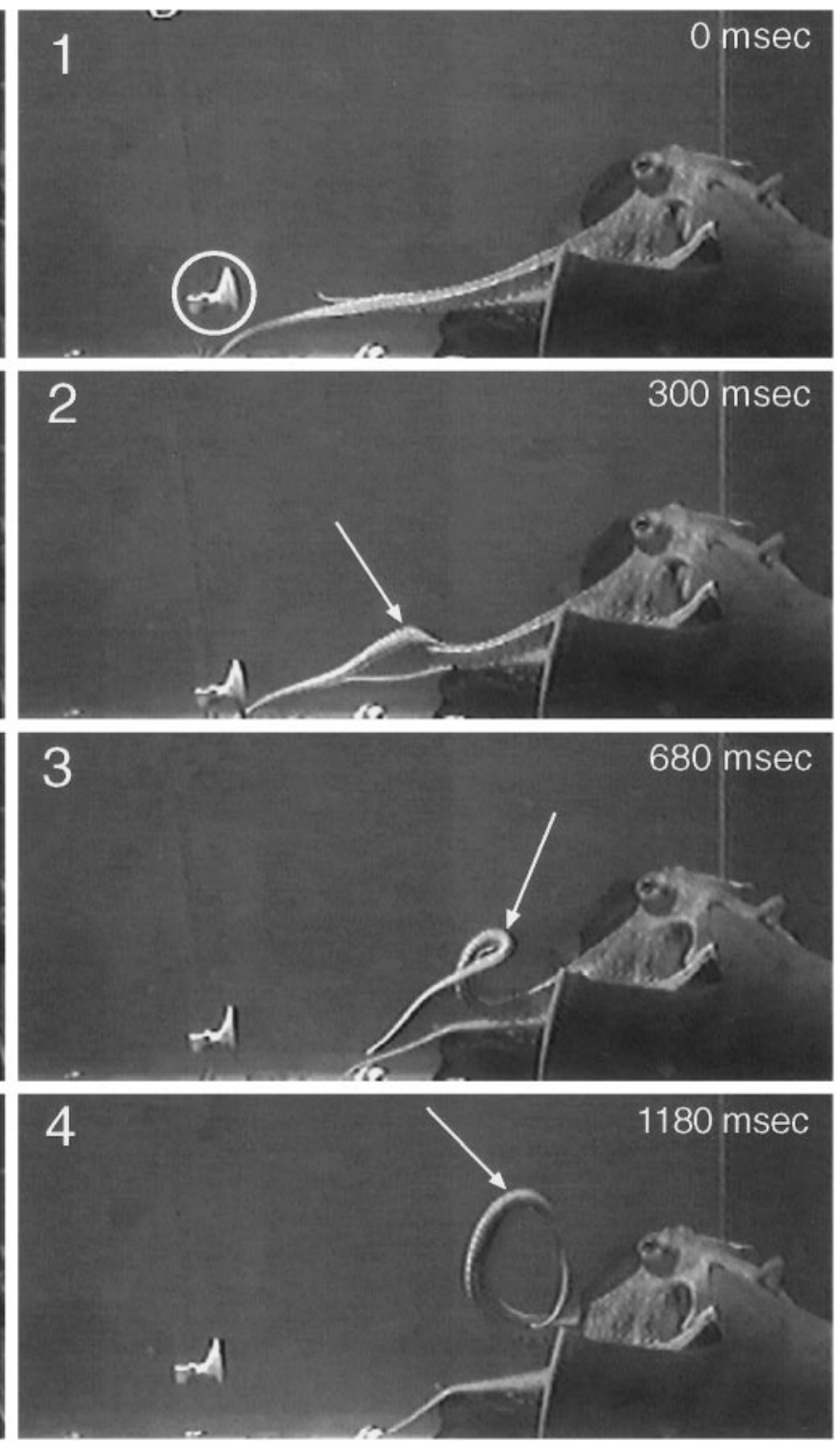

Figure 1. An example of two stereotyped motor patterns: bend propagation $(A)$ and bend initiation $(B)$. Four video frames taken during the course of the movement are presented for each example (1-4). Time in milliseconds is given in the top right corner of each frame. The target, a green plastic disk (marked by a circle in $A 1$ and B1), is in the bottom left corner. The arrowhead in $A 1$ shows the sucking rings. Notice the bend in the arm (arrow in $A$ ), which propagates toward the tip. In an already extended arm (B1), a bend is initiated at a certain point along the arm (arrow in B2), and the part distal to this point is pulled backward $(B 3-4)$.

is rigid give a good estimation of the movement of the body center. To characterize the kinematic features of arm extension independent of body movements, we have analyzed the movement of the bendpoint relative to the body. The relative movement was obtained by vectorially subtracting the measured movement of the eye from the bend-point movement. An example of the subtraction is depicted in Figure $2 B$. This procedure was repeated in all trials.

\section{Bend-point path}

Analysis of the bend-point path in 84 reaching movements shows that the bend-point tends to move within a single plane in a simple, slightly curved path. The plane of motion and the degree of planarity were determined by multiple linear regression (see Materials and Methods). Table 1 summarizes the average distance traveled by the bend-point in the plane of motion, the average SE of the fit (residual error), and the average $R^{2}$. In all trials (except one in octopus C), $R^{2}$ was significantly positive ( $p<0.01$; F-test). The average SE is small compared with the average movement in the plane of motion. This, as well as the high average $R^{2}$ values, indicates that to a good approximation the bend-point moves within a single plane. In general, this plane corresponds to the sagittal plane of the arm, which is defined by the ventral location of the sucking rings.

The planarity of the movement enables us to reduce the space of interest to two dimensions without losing significant information. Figure $3 A$ shows the data from Figure 2 in a transformed coordinate system where the $X Z$ plane is parallel to the best fitted 


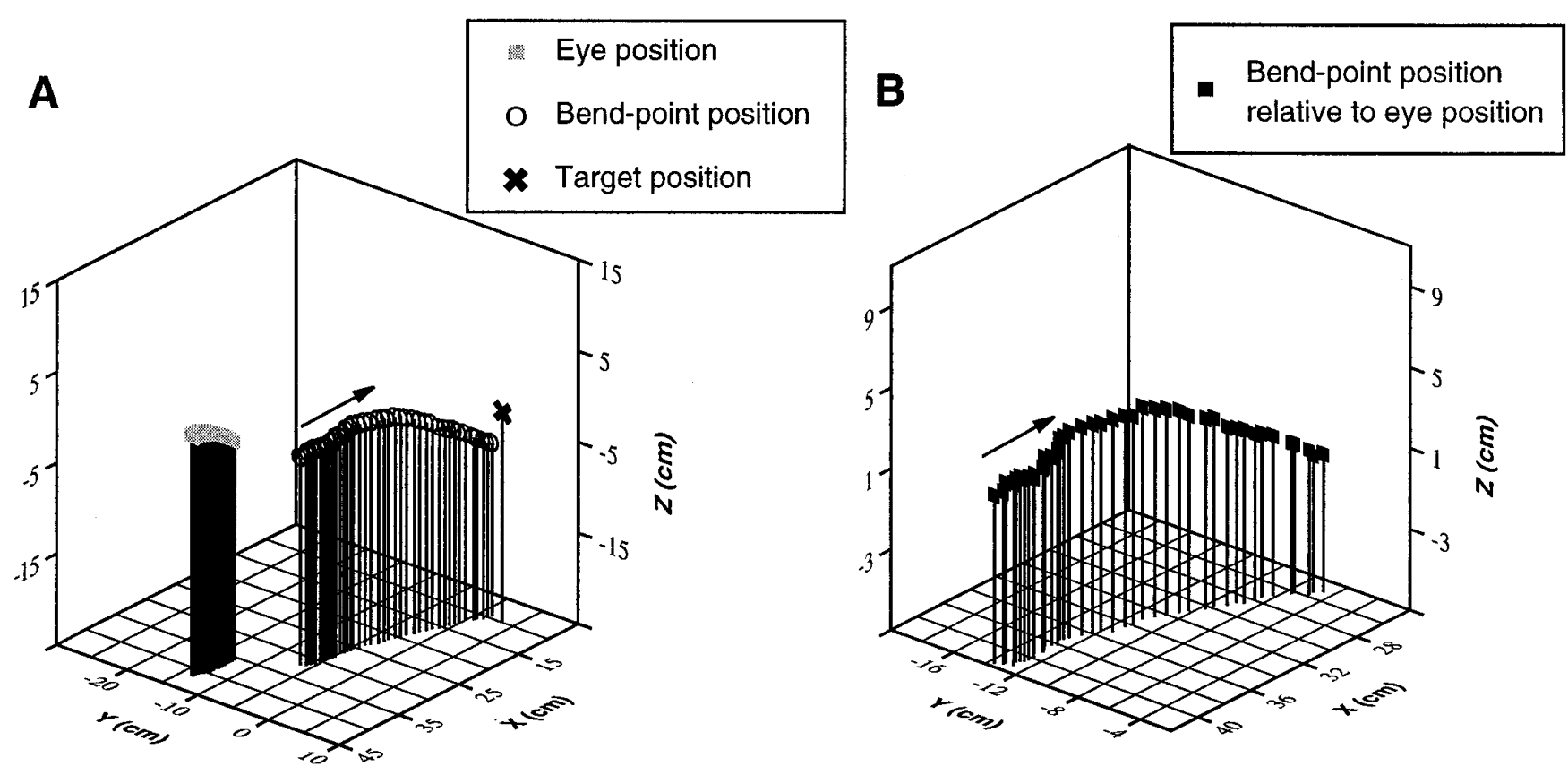

Figure 2. Movement of the bend-point (the midpoint of the bend in the arm) in 3D space. The DLT algorithm (see Materials and Methods) for analyzing stereo video recordings was used to obtain a description of the movements in space and time. $A, 3 \mathrm{D}$ graph showing eye (rectangle) and bend-point (circles) position during a reaching task. Target location is marked by an $X$. Each point on the graph represents the position in a single video field (20 msec interval between images). The arrow marks the direction of movement. $B$, The movement of the bend-point relative to the eye. The direction of movement is marked by the arrow.

plane. As expected from a planar movement, the data points in the two-dimensional view of the transformed $Y Z$ plane are organized along a straight line (Fig. $3 B$ ).

The characteristic features of the bend-point path within the best fitted plane are shown in Figure $4 A-C$ for the three animals tested. Four different reaching movements are shown for each animal. The dots mark the data, and the lines mark the fitted fifth-order polynomials. The direction of movement is indicated by the arrows. In all animals the path is either slightly curved or, less frequently, nearly a straight line. These results indicate that the bend-point moves in a relatively simple path in respect to the animal's body.

\section{Bend-point velocity}

The tangential velocity of the bend-point as a function of time (velocity profile) during a reaching task was calculated from 84 trials (see Materials and Methods). It should be noted that a bend is a dynamic structure that can evolve or disappear during the movement of the arm, and so the velocity may start and end at values other than zero, as can be seen in Figure $5 A$. This contrasts with measurements of the velocity of fixed points in moving structures, like the tip of the arm (Hollerbach and Flash, 1982).

Table 1. Summary for three octopuses showing the average SE of the fit of the bend-point path to the plane of motion, the average distance traveled by the bend-point in the plane of motion, and the average $R^{2}$

\begin{tabular}{lccc} 
& Octopus A & Octopus B & Octopus C \\
\hline SE & $0.60 \pm 0.25 \mathrm{~cm}$ & $0.42 \pm 0.16 \mathrm{~cm}$ & $0.49 \pm 0.19 \mathrm{~cm}$ \\
Distance & $33.44 \pm 12.3 \mathrm{~cm}$ & $25.80 \pm 8.9 \mathrm{~cm}$ & $29.74 \pm 8.1 \mathrm{~cm}$ \\
$\mathrm{R}^{2}$ & $0.88 \pm 0.10$ & $0.89 \pm 0.14$ & $0.75 \pm 0.24$ \\
Number of trials & 33 & 29 & 22
\end{tabular}

Values are averaged over all trials with \pm SD.
Figure $5 A$ shows three velocity profiles measured from the same octopus. Although the range of velocities of these movements varies, they all demonstrate a characteristic velocity profile. This profile can be divided into three phases (Fig. $5 A$, arrows in curve $c)$. Phase I is the initial part of the movement. This phase ends at the minimum (marked by the left arrow) and is characterized by a monotonic decrease in velocity (profiles $a$ and $c$ ) or by an initial increase in velocity followed by a local peak (profile $b$ ). Phase II, which starts at the local minimum and ends at the peak velocity (right arrow), is characterized by a monotonic increase in velocity. Phase III is the final phase of the movement where the velocity decreases until the bend-point disappears.

Phase I corresponds to the transition between the initiation and propagation of the bend (Fig. $1 B$ ). This stage of the movement is variable; it depends on the initial position of the arm and on the direction of arm extension. Phase II corresponds to the propagation of the bend-point along the arm. This stage, which is the most prominent and robust, is shown in Figure $1 A$. The main part of the arm extension occurs during this stage. Typically, the velocity reaches a maximum in the vicinity of the target. After this maximum, the bend moves toward the tip with what seems to be a passive wave propagation. This stage corresponds to the decrease in velocity in phase III. In many cases, the bend disappears before reaching the tip of the arm. This basic velocity pattern was observed in 65 of the 84 tangential velocity profiles. The remaining 19 cases, which were distributed among the three animals, did not match this basic pattern and demonstrated various velocity profiles.

Invariance of tangential velocity is commonly tested by normalizing the movement speed with respect to amplitude and duration (Atkeson and Hollerbach, 1985). A major problem in using this method in the study of the movement of the bend in the octopus arm is the uncertainty in determining the beginning and end of the 


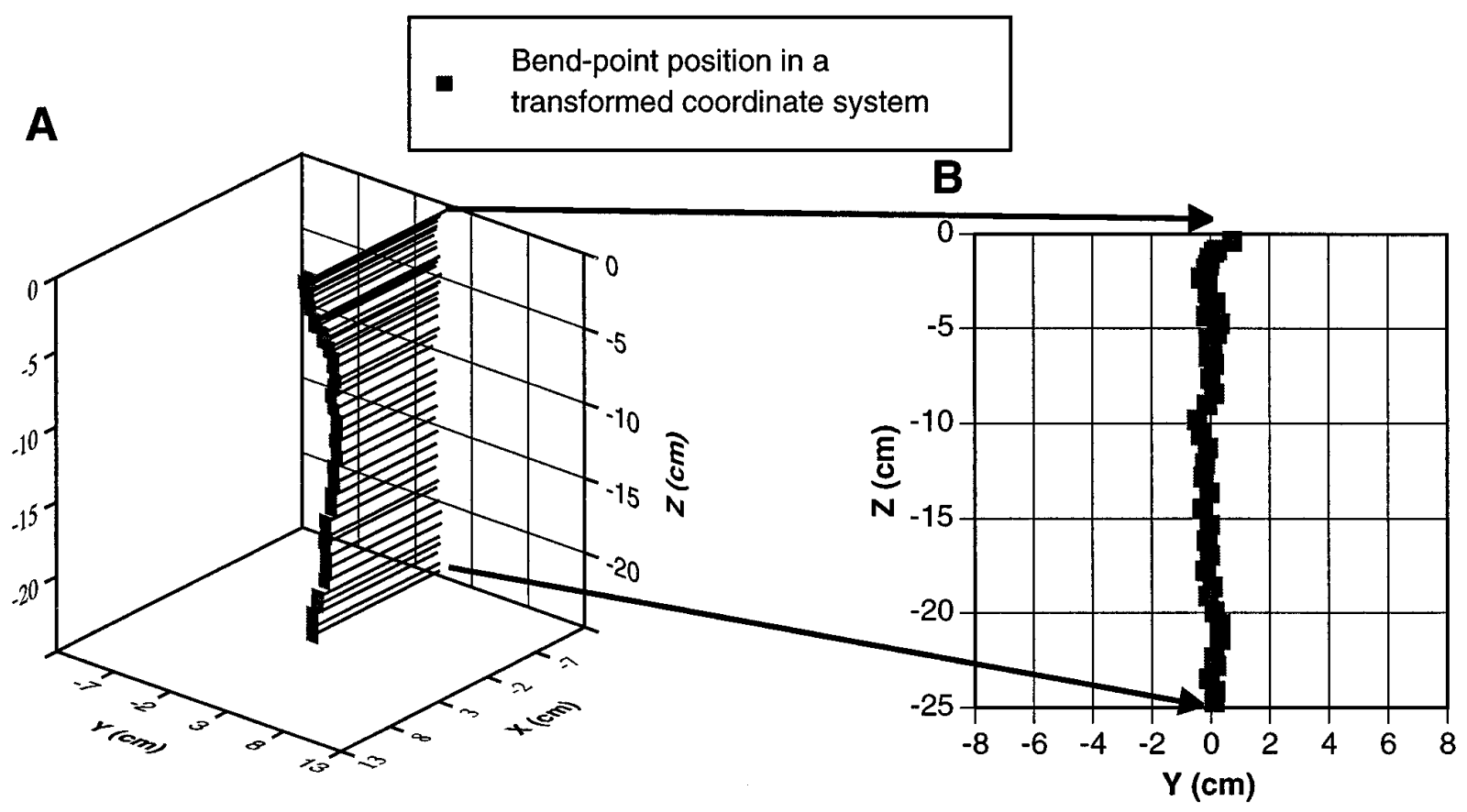

Figure 3. Bend-point path is planar. $A, 3 \mathrm{D}$ graph showing the data from Figure $2 B$ in a transformed coordinate system where the $X Z$ plane is parallel to the plane of best fit. $B, 2 \mathrm{D}$ view of the $Y Z$ plane, demonstrating the planar organization of the movement. In the example shown, the SE of the fit is $0.61 \mathrm{~cm}$.

movement. This uncertainty arises from (1) the nature of this movement, which as mentioned above can begin and end with various velocities, and (2) the fact that we are studying movements of naturally behaving animals. The latter, in contrast to studies of a controlled movement in humans or in well trained animals, imposes various initial and terminal conditions. To overcome this problem, we took advantage of the fact that the tangential velocity profiles of the octopus arm extensions almost always followed a profile with a well defined minimum and maximum (marked by the arrows in Fig. $5 A$ ). We therefore normalized the velocity $[V(t)]$ and the time $(t)$ according to the maximum velocity $\left(V_{\max }\right)$ and the distance $(D)$ traveled by the bend-point between these minima and maxima as follows:

$$
\begin{gathered}
V_{\text {normalized }}=V(t) / V_{\max } \\
T_{\text {normalized }}=V_{\max } \times t / D .
\end{gathered}
$$

$D$ was calculated using:

$$
D=\sum_{t} \sqrt{\left(X_{t}-X_{t-1}\right)^{2}+\left(Z_{t}-Z_{t-1}\right)^{2}}
$$

where $X$ and $Z$ are the coordinates of the smoothed data in the best fitted plane, and the index $t$ designates the image number (time).

In Figure $5 B$, the three profiles from Figure $5 A$ have been normalized and superimposed by aligning the peaks. These normalized curves demonstrate a clear overlap in phase II, suggesting that some common constraint dictates the pattern of velocity increase during this phase. To examine the generality of this observation, all velocity profiles with the characteristic pattern as shown in Figure $5 A$ were superimposed in Figure $6 A-C$. In addition, an average velocity profile and its variance were calcu- lated for each animal (Fig. 6D). The superimposition of the normalized velocities (Fig. $6 A-C$ ) shows that the result of Figure $5 B$, in which phase II is invariant and phases I and III are variable, holds for the majority of arm extensions. The similarity of the velocity profiles during phase II is demonstrated further by the variance shown in Figure $6 D$ : the variance during phase II is very low, relative to the variance during phases I and III. Velocity profiles are similar, not only in individual animals but also among different animals, as shown by the remarkable overlap of the average normalized velocity profiles from different animals (Fig. 6D).

\section{Two-arm coordination}

The issue of coordination between different arms is especially interesting in the case of the octopus, because of its need to control eight flexible arms. An insight into the mechanism of arm coordination can be obtained from those trials in which the octopus extends two or more arms. Figure 7, $A$ and $B$, shows two examples where the octopus extends two arms toward the target (located in the bottom left corner of the picture) by propagating a bend in each of the arms (marked by arrows). The arms can either move together, as shown in Figure $7 A$, or move one after the other, as shown in $7 B$. We have analyzed the velocity of the bend-point propagation for the cases of synchronous and consecutive movements. Figure $7 C$ shows the bend-point positions of two arms moving simultaneously; the tangential velocities are depicted in $7 D$. Note that the curves in Figure $7 D$ are not normalized. It is clear that the velocity of both arms follows a similar pattern. Such velocity coupling was also observed in arms moving consecutively with a short delay between them. An example is shown in Figure $7, E$ and $F$. In this trial, the octopus first extended one arm toward the target, and $1.3 \mathrm{sec}$ later a second arm was extended (bend-point positions are shown in $E$ ). 

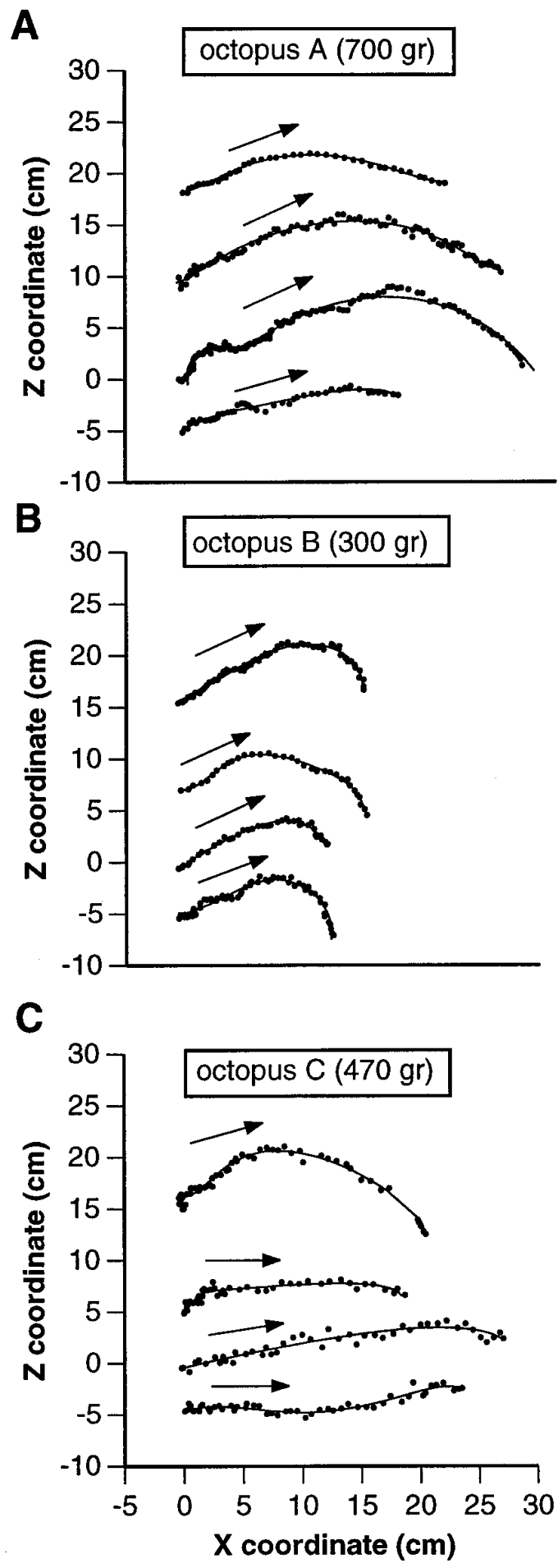

Figure 4. Examples of the bend-point path measured in different trials and from different octopuses. The path of four different reaching movements is depicted for each animal $(A-C)$ on the plane of movement (the best-fit linear plane). The dots show the measured positions, and the lines are the fitted fifth-order polynomials (see Materials and Methods). The direction of movement is indicated by the arrows. The initial point of the movements was displaced on the graph to improve clarity of display.

Clearly, in this case the two arms also moved with almost identical velocity profiles but with a constant phase lag of $\sim 1.3$ sec between them $(F)$.
To quantify the similarities between the velocity profiles of two moving bend-points, we first aligned the profiles at the beginning of the movement and then calculated the RMS of the velocity differences according to:

$$
\mathrm{RMS}=\sqrt{\frac{\sum_{t=0}^{n}\left(V_{1 t}-V_{2 t}\right)^{2}}{n}},
$$

where $V_{1}$ and $V_{2}$ are the velocities of the two bend-points, $t$ is the image number (20 msec interval), and $n$ is the number of images where the velocity was measured and compared. This procedure was repeated for three groups of movements: (1) synchronous movements, where the two arms moved toward the same target at the same time; (2) consecutive movements, where the arms moved to the same target (same trial) but with a short delay between them $(0.4-1.3 \mathrm{sec})$; and (3) control group movements. For this group we arbitrarily chose movements from two different trials performed by the same octopus. The average RMS of these three groups is shown in Table 2 .

The similarity between the velocity profiles in the synchronous group, as well as for the consecutive group, is significantly larger than for the control group ( $p<0.01$; Mann-Whitney rank test). We conclude that when two arms are moving toward the same target, they tend to move with the same velocity profile. This result suggests a common source for the generation of movements that are produced within the same behavioral event.

\section{Bend propagation in spontaneous movements}

As mentioned above, the generation and propagation of a bend occurs during various behaviors. This raises the question of whether the same stereotyped characteristics of bend-point path and velocity as seen in the reaching task are also to be found during other behaviors. To answer this question, we compared the trajectories generated in spontaneous movements (those not directed toward a target) with those measured during reaching movements.

Bend propagation in spontaneous movements occurs either when the arm is moving freely in the water (unconstrained movements) or when the arm is extended along a solid surface such as the aquarium side (constrained movements). In the latter case, the bend travels on the surface while the sucking rings proximal to the bend grip this surface. We examined bend-point trajectories from both of these modes of movement (unconstrained and constrained).

The spontaneous movements were analyzed using the same analytical procedure as for the reaching movements. First, the planarity of the movement was tested by calculating the best fit linear plane containing the movement. This part of the analysis was applied only to unconstrained movements, because the con-

\begin{tabular}{|c|c|c|}
\hline & $\begin{array}{l}\text { Average RMS } \pm \\
\mathrm{SD}(\mathrm{cm} / \mathrm{sec})\end{array}$ & Number of pairs \\
\hline \multicolumn{3}{|l|}{ Synchronous } \\
\hline group & $6.26 \pm 1.7$ & 10 \\
\hline Consecutive group & $6.84 \pm 2.5$ & 7 \\
\hline Control group & $13.46 \pm 5.1$ & 10 \\
\hline
\end{tabular}


A

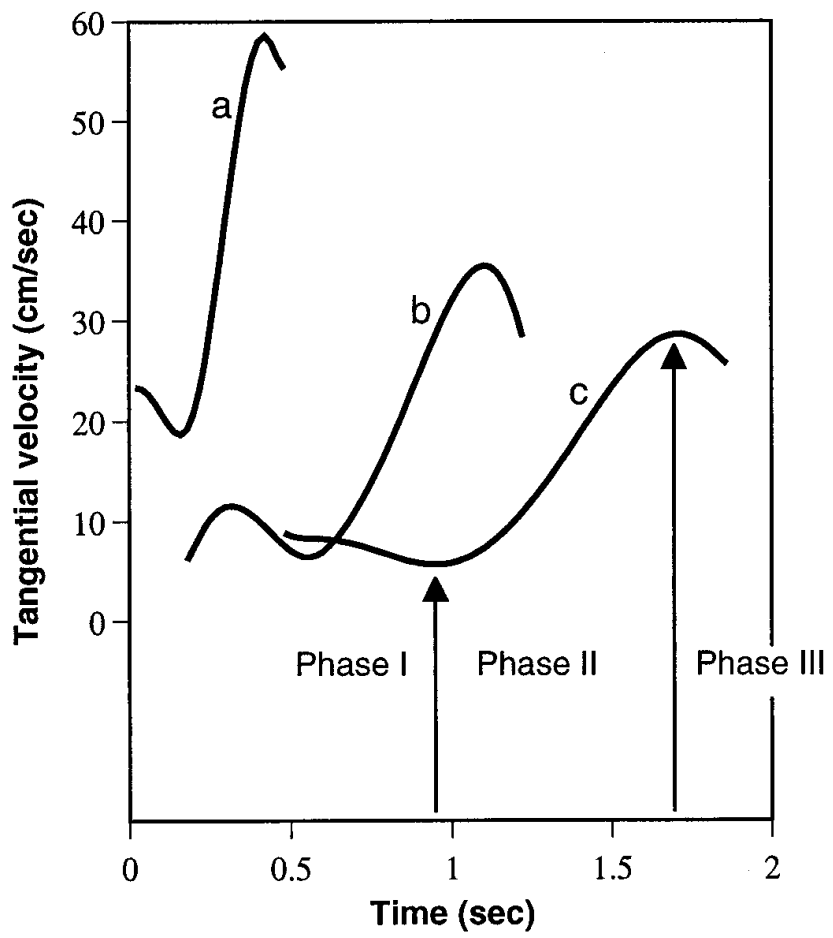

B

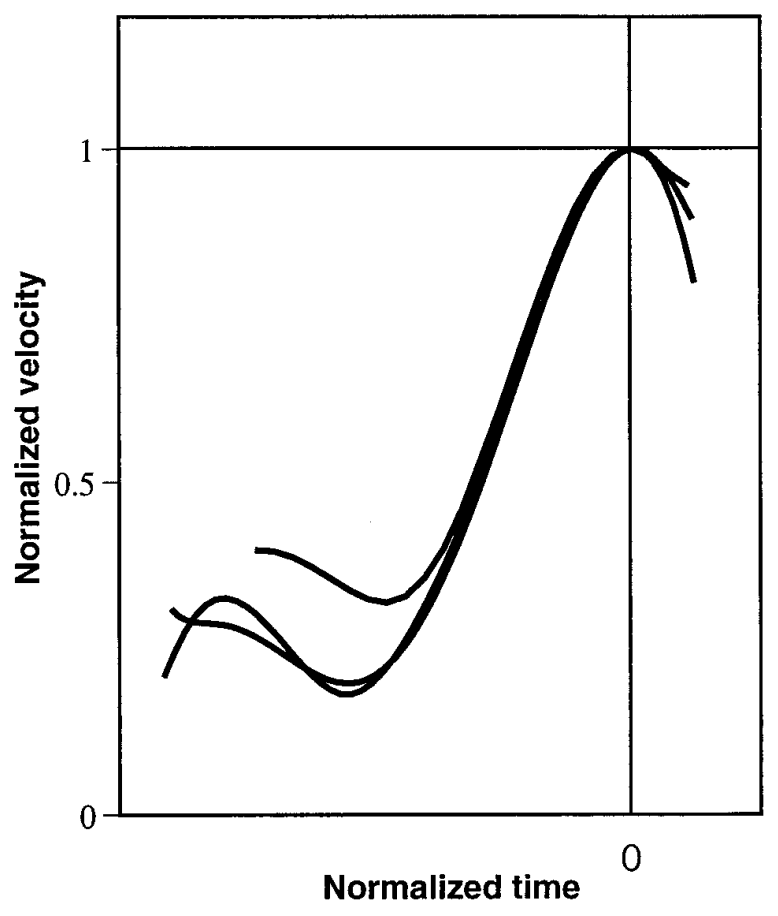

Figure 5. Tangential velocity profiles are invariant. $A$, Bend-point tangential velocity, calculated from the smoothed kinematic data during three trials, is plotted against time. The profiles share a similar pattern, which can be divided into three phases (marked by the arrows). This division corresponds to different stages of the movement (see Results). $B$, The velocity profiles shown in $A$, normalized for velocity and distance and aligned at the peak, demonstrate a substantial overlap during phase II.

strained movements are limited a priori to a surface. We found that the paths of the bend-points in the unconstrained movements were confined to a single plane (average $R^{2}$ of $0.81 \pm 0.19 ; n=11$, all statistically significant). This was similar to the results obtained in the reaching movements (Table 1).

In 10 of the 11 unconstrained movements measured, the tangential velocity profiles had a characteristic pattern similar to that shown in reaching behavior (compare Fig. $8 A$ with Figs. 5 and 6). In Figure $8 C$, the four tangential velocity profiles from Figure $8 A$ (solid lines) have been normalized and superimposed together with three normalized velocity profiles measured during a reaching task (dashed lines). The significant overlap of all the curves demonstrates that the invariant nature of the velocity profiles shown in reaching movements is found in spontaneous unconstrained movements as well.

In contrast, six of eight constrained movements did not resemble this basic pattern. Four examples are shown in Figure $8 B$. Their velocity profiles showed either constant velocity (two lower traces) or slight changes in speed. The constrained movements had generally lower velocities.

\section{DISCUSSION}

This study is the first detailed and quantitative kinematic analysis of octopus arm movements. It shows that despite the fact that an octopus arm has virtually infinite degrees of freedom, arm movements are executed in a stereotyped manner. Our study has focused on extension movements that are generated by a bend propagating along the arm. This bend propagation is a basic movement pattern that is used in different behaviors, such as locomotion, searching, and reaching.
In flexible structures, a propagating bend can be generated by either a passive whip-like action or an active mechanism involving muscle contraction along the arm. We have shown previously that bend propagation in the octopus arm is associated with a propagating wave of muscle activity (Hochner et al., 1995), indicating an active mechanism. According to the constant volume constraint for muscular-hydrostats, contraction of the longitudinal muscles on one side of the arm and simultaneous activation of the transverse muscles, to resist increase in arm diameter, should result in the formation of a bend (Kier and Smith, 1985). Hence, a propagating bend would be generated by a wave of coordinated local contractions. It is also possible that after the initiation of a bend, its propagation is a result of a stiffening wave that propels the bend toward the tip of the arm. This issue is currently under investigation.

The present kinematic study demonstrates that in the octopus arm, the bend tends to propagate along a stereotyped trajectory. In particular, the bend propagates along a relatively simple curved path, which is contained in a linear plane. Although the path itself is not necessarily invariant, the normalized velocity profile has the same kinematic form regardless of movement direction, speed, or amplitude. This observation resembles results obtained from human and primate arm movement studies (Morasso, 1981; Abend et al., 1982; Hollerbach and Flash, 1982; Atkeson and Hollerbach, 1985).

Is there a general movement-generation principle that is common to both cases? Octopus arms are essentially different from human-like arms in both anatomy and control mechanisms. The aim of the control system in human-like arms is to bring the tip of 

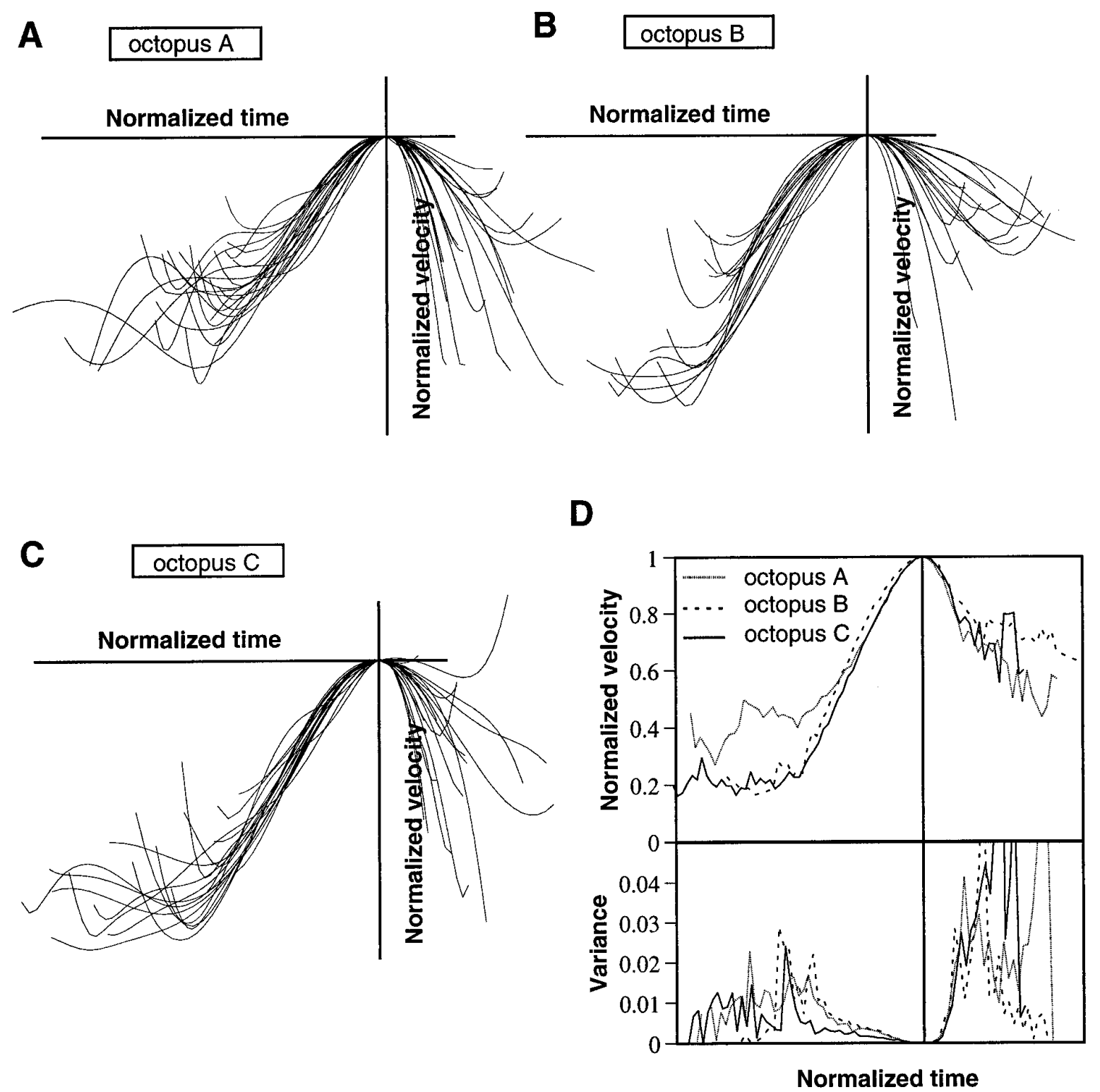

Figure 6. Comparison between normalized tangential velocity profiles from different animals. Sixty-five normalized velocity profiles aligned at the peak are displayed in three graphs $(A-C)$ corresponding to three animals. An average velocity profile and its variance were extracted for each animal and superimposed in $D$. A remarkable similarity of the average normalized velocity profiles from the different animals is observed $(D$, top graph).

the arm to the target, whereas in octopus any part of the arm can be used to grab the target. There is, therefore, no special significance to the tip point or any other fixed point along the arm; however, the bend is instrumental in leading the arm in the desired direction. In this sense, like the human hand, controlling the bend-point trajectory is a reasonable strategy. We have shown that the bend-point follows a simple planar path from the body center toward the target. The simplicity and stereotyped appearance of this path suggests that bend-point position is an important variable for the planning and control of octopus arm movements. Another possibility, however, is that the simple and stereotyped appearance is a by-product of some unknown constraint in the system. In this case the bend-point per se would not be controlled directly by the nervous system.

Researchers of motor control of articulated arms have pointed out the trade-off between planning movement in external coordi- nates and the computational difficulties of executing the planned movement (Hollerbach, 1990b; Flanders et al., 1992; Haggard et al., 1995). Planning in terms of the external coordinates of the tip of the arm allows the external constraints to be dealt with more easily. Executing a movement planned in this way, however, raises computational difficulties because of the complex transformations between end-point coordinates, joint coordinates, and muscle activity (Bizzi, 1993; Gielen, 1993). The complexity of such transformations, which are termed the "inverse problems," arises mainly from the excess degrees of freedom of the limb compared with the degrees of freedom of the end effector or the task (Bizzi et al., 1991).

What is the analogy for the inverse problem in octopus arm movements? If bend-point location in time and space is an important controlled variable, the inverse problem in the octopus is the transformation from bend-point coordinates to muscle activ- 
A

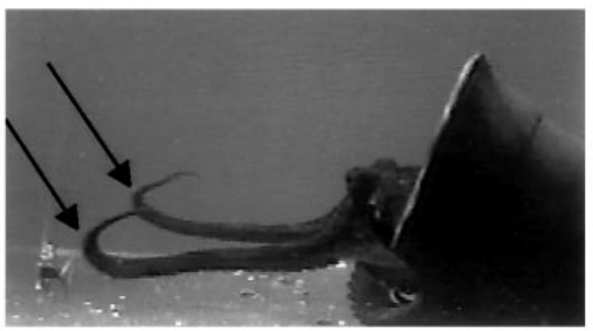

B

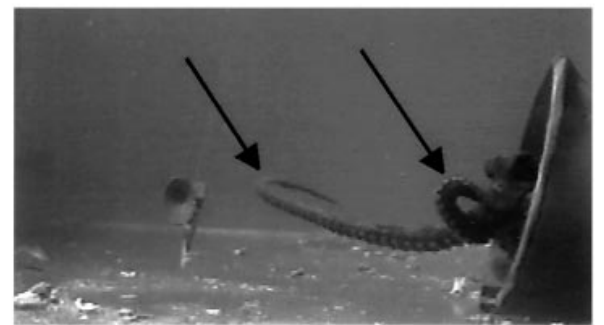

Two arms moving synchronously
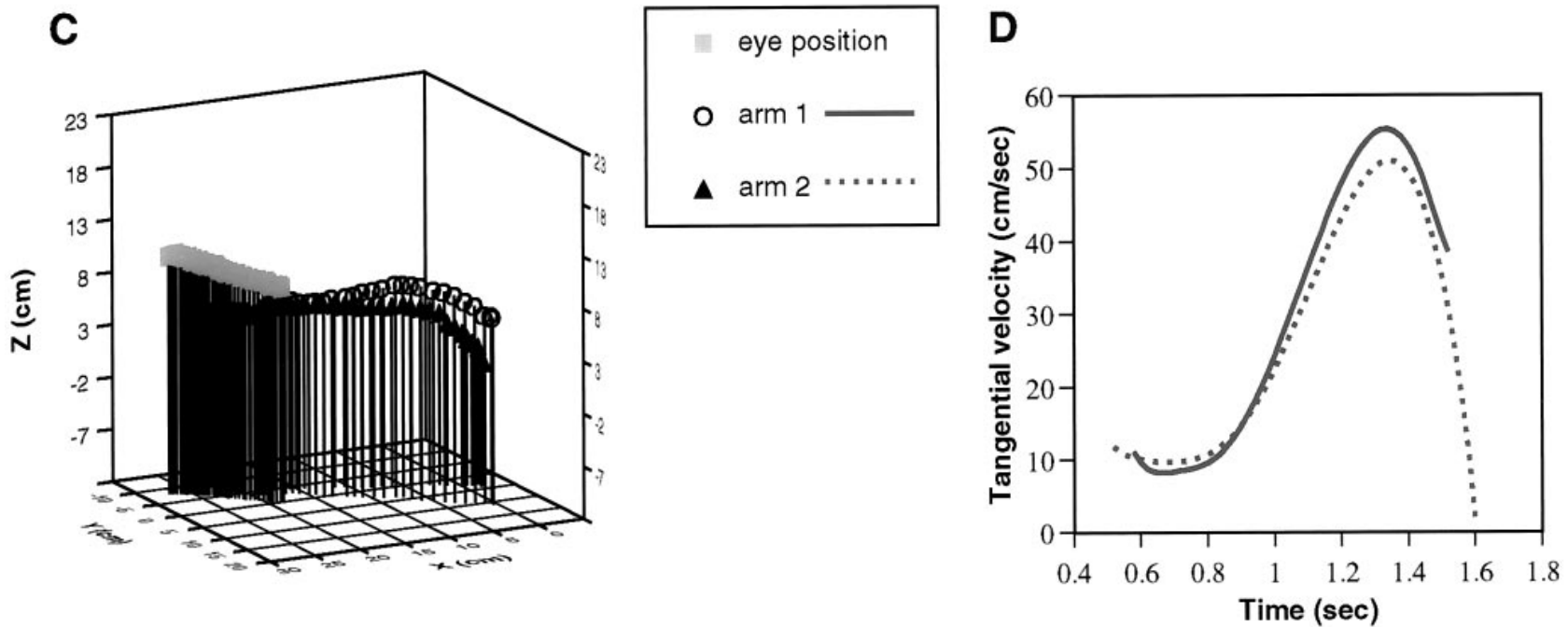

$1.3 \mathrm{sec}$ delay between the arms

E

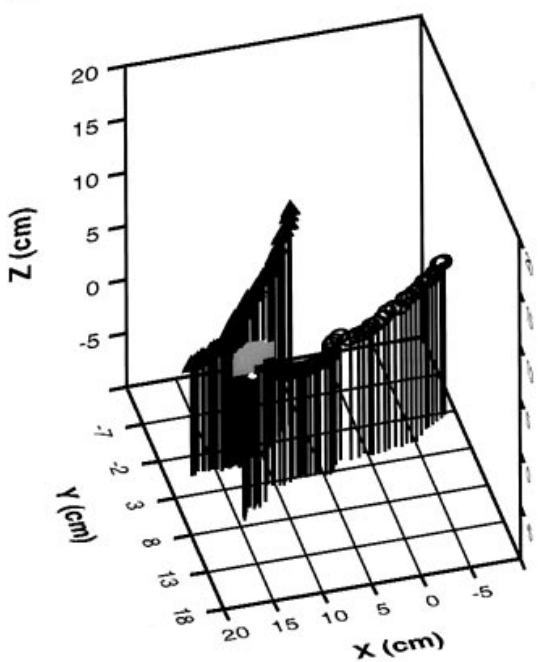

$\mathbf{F}$

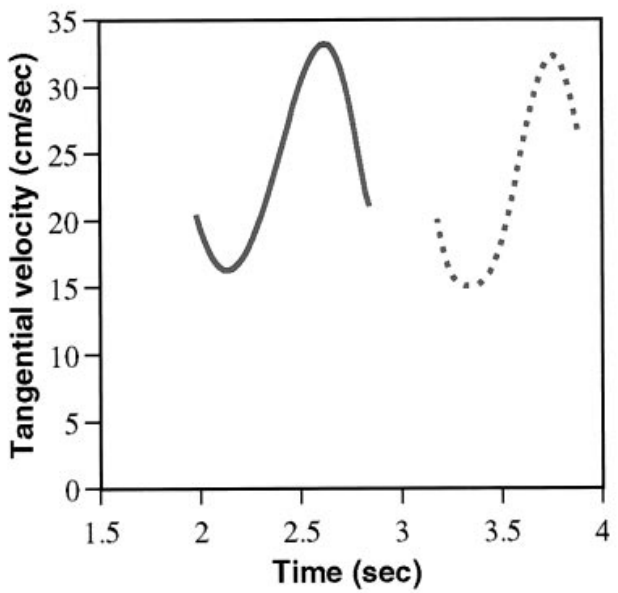

Figure 7. Two arms moving toward a target simultaneously, or one after the other with a short delay, tend to move at the same speed. $A, B$, Video frames showing an octopus extending two arms toward the target, which is in the left side of the frame. $A$, Two arms moving simultaneously. $B$, Two arms moving one after the other. $C, D$, Bend-point trajectories of two arms moving simultaneously were measured. The positions of the bend-points and eye are shown in $C$. The tangential velocity profiles $(D)$ follow a similar pattern. $E, F$, Bend-point trajectories from two arms moving one after the other with a short delay. The path of the two arms is shown in $E$, and the tangential velocities plotted against time are shown in $F$.

ity. This transformation, however, need not be that complicated. Assuming that only one bend is traveling along the arm, and indeed this is the case in the reaching task, the number of degrees of freedom of the arm can be reduced dramatically: one for the movement of the bend along the axis of the arm and two for the yaw and pitch movements of the arm around its base (roll move- 
A

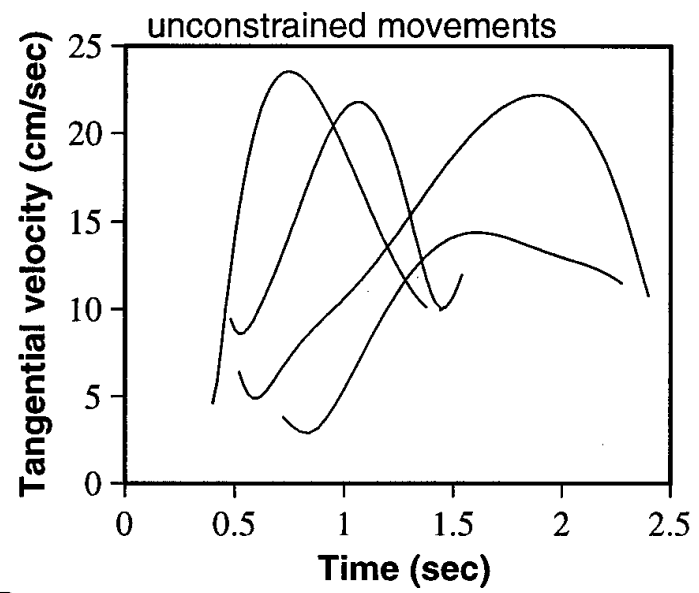

B

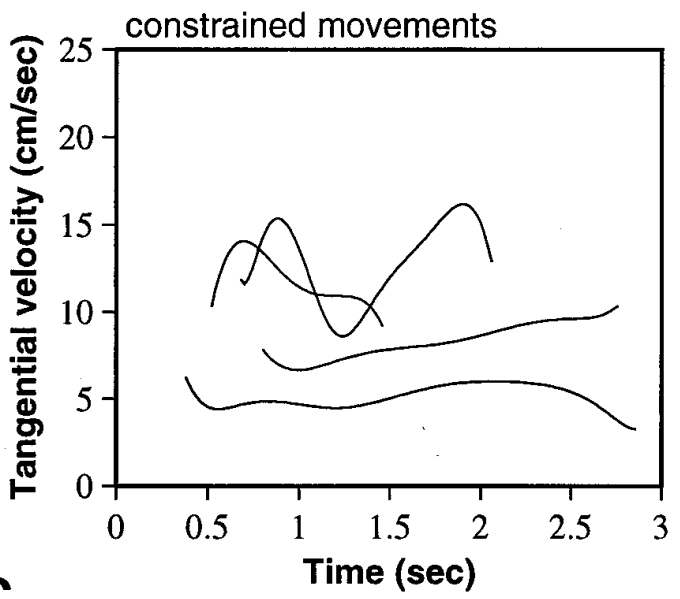

C

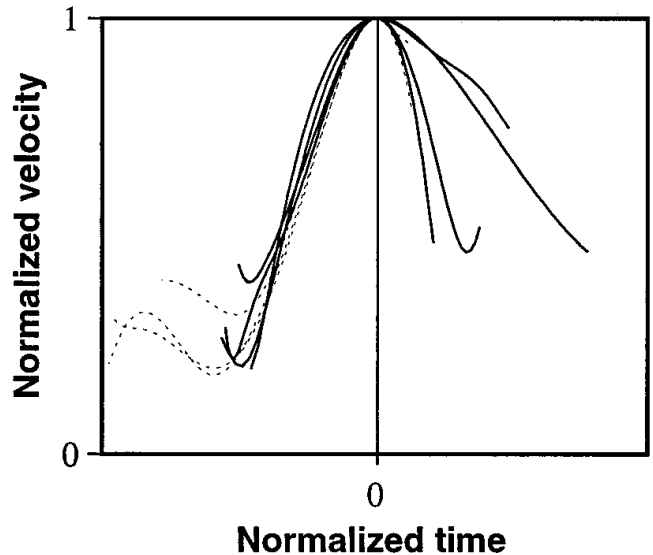

Figure 8. Tangential velocity profiles measured from spontaneous arm extensions. $A$, Four tangential velocity profiles measured during spontaneous unconstrained movements (i.e., movements freely in the water and not directed toward a target). $B$, Four tangential velocity profiles measured during constrained spontaneous movements (touching the surface of the aquarium walls or floor). $C$, The normalized velocity profiles of the unconstrained movements shown in $A$ (solid lines) are superimposed on three normalized velocity profiles obtained from movements during a reaching toward a target (dashed lines).

ments are not observed during reaching). Such a system reduces computational complexity tremendously. Moreover, because bend propagation is generated by a propagating wave of muscle con- traction (Hochner et al., 1995), the position of the bend along the arm and muscle activity share the same coordinates, allowing relatively simple transformations between the planned bend-point movement and the required muscle activation. Therefore, assuming that inverse computation takes place in this system, it is plausible that this basic motion pattern has evolved not only as a result of biomechanical constraints or motor objectives (such as energy minimization or maximizing motion smoothness) but also as a result of the need to minimize computational complexity.

We have found that different reaching movements vary in speed and distance but maintain a basic velocity pattern repeated in both target-oriented and spontaneous movements. Furthermore, the same pattern is observed in different octopuses. The particular shape of the velocity profile (tangential velocity of the bend as a function of time) may reflect an optimal neuronal control strategy or might be attributable to some mechanical factors. We found further that the mid-part of the movements (i.e., phase II in Fig. 5) can be attributed to the same basic profile scaled for speed and distance. One explanation for the scaling properties is that bend propagation is generated by a built-in motor program that can be adjusted by simple scaling of the same pattern to produce different speeds. The output of such a system is a basic invariant trajectory that reflects the dynamics of the underlying neural activation pattern (Gracco, 1988; Smith et al., 1995). Because bend propagation is a common movement frequently repeated in the course of different motor behaviors, it is plausible that a stored neuronal pattern coordinates muscle contraction to generate a propagating bend along the arm. We propose that this pattern can be simply modified to produce different velocities. Support for this idea is provided by the results of the tangential velocity profiles of two arms moving together. Two arms moving synchronously, or at a short time lag, generate similar velocity profiles with the same peak velocity. This result suggests that the movement is generated by a similar motor command at the neuronal level and that this command is stored, at least for short times, in the control system. Furthermore, the fact that the same bend-point trajectories are measured in unconstrained spontaneous movements as well as in movements toward a target suggests that a common movement source or pattern generator produces bend propagation in these two different behavioral contexts.

A number of issues, however, still remain unclear. What is the explanation for the variance observed in the scaled velocities and in the path of the bend-point? Why is the basic pattern not demonstrated in all reaching movements? The nervous system of the octopus is divided into a central brain and axial nerve cords along the arms. The majority of the nerve cells are located in those axial nerve cords (Young, 1971). The muscles of the arm are innervated by $\sim 3.8 \times 10^{5}$ fibers, which originate from neurons within the arm. They are controlled by the brain via only 4000 efferent nerve fibers. Sensory information is gathered by some $2.3 \times 10^{6}$ receptors but only $\sim 17,500$ sensory nerve fibers reach the brain from each arm (Young, 1965). These numbers indicate the extensive role of the local neural circuitry in controlling the behavior of the arm, and indeed, various local reflexes involving chemical and touch sensation have been reported in the octopus arm (Wells and Wells, 1957; Rowell, 1966; Wells, 1978; Altman, 1971). Thus, it is most reasonable to assume that the movement of the arm is the outcome of both preplanned feedforward central commands and interactions with the environment through the local sensorimotor circuits. It is possible that deviations from the basic movement pattern observed in the present experiments reflect 
adjustment of the neuronal command according to incoming sensory input. Indeed, stereotyped tangential velocity profiles were abundant in the unconstrained movements, whereas in the constrained movements, where sensory input from the arm is probably dominant in shaping the movement, such an invariant form of the velocity profiles was not observed.

\section{REFERENCES}

Abend W, Bizzi E, Morasso P (1982) Human arm trajectory formation. Brain 105:331-348.

Altman JS (1971) Control of accept reject reflexes in the octopus. Nature 229:204-206.

Atkeson CG, Hollerbach JM (1985) Kinematic features of unrestrained vertical movements. J Neurosci 5:2318-2330.

Bizzi E (1993) Intermediate representations in the formation of arm trajectories. Curr Opin Neurobiol 3:925-931.

Bizzi E, Mussa-Ivaldi FA, Giszter S (1991) Computations underlying the execution of movement: a biological perspective. Science 253:287-291.

Chiel HJ, Crago P, Mansour JM, Hathi K (1992) Biomechanics of a muscular hydrostat: a model of lapping by a reptilian tongue. Biol Cybern 67:403-415.

Fiorito G, Planta CV, Scotto P (1990) Problem solving ability of Octopus vulgaris Lamarck (Mollusca, Cephalopoda). Behav Neural Biol 53:217-230.

Flanders M, Tillery SIH, Soechting JF (1992) Early stages in sensory motor transformations. Behav Brain Sci 15:309-362.

Flash T, Hogan N (1985) The coordination of arm movements: an experimentally confirmed mathematical model. J Neurosci 5:1688-1703.

Gielen SC (1993) Movement dynamics. Curr Opin Neurobiol 3:912-916.

Gracco VL (1988) Timing factors in the coordination of speech movements. J Neurosci 8:4628-4639.

Haggard P, Hutchinson K, Stein J (1995) Patterns of coordinated multijoint movement. Exp Brain Res 107:254-266.

Hochner B, Gutfreund Y, Fiorito G, Flash T, Segev I, Yarom Y (1995) Arm electromyograms in freely moving octopus (Octopus vulgaris). Isr J Med Sci 31.12:745.
Hollerbach JM (1990a) Fundamentals of motor behavior. In: Visual cognition and action (Osherson DN, Kosslyn SM, Hollerbach JM, eds), pp 151-182. Cambridge: MIT.

Hollerbach JM (1990b) Planning of arm movements. In: Visual cognition and action (Osherson DN, Kosslyn SM, Hollerbach JM, eds), pp 183212. Cambridge: MIT.

Hollerbach JM, Flash T (1982) Dynamic interactions between limb segments during planar arm movement. Biol Cybern 44:67-77.

Kier WM (1982) The functional morphology of the musculature of squid (Loliginidae) arms and tentacles. J Morphol 172:179-192.

Kier WM, Smith KK (1985) Tongues, tentacles and trunks: the biomechanics of movement in muscular-hydrostats. Zool J Linn Soc 83:307-324.

Morasso P (1981) Spatial control of arm movements. Exp Brain Res 42:223-227.

Press WH, Teukolsky SA, Vetterling WT, Flannery OP (1992) Numerical recipes in C: the art of scientific computing. Cambridge, UK: Cambridge UP.

Rowell CHF (1966) Activity of interneurons in the arm of octopus in response to tactile stimulation. J Exp Biol 589-605.

Smith A, Goffman L, Zelaznik HN, Ying G, McGillem C (1995) Spatiotemporal stability and patterning of speech movement sequences. Exp Brain Res 104:493-501.

Smith KK, Kier WM (1989) Trunks, tongues, and tentacles: moving with skeletons of muscle. Am Sci 77:29-35.

Wells MJ (1978) Octopus. London: Chapman and Hall.

Wells MJ, Wells J (1957) The function of the brain of octopus in tactile discrimination. J Exp Biol 34:131-142.

Woltring HJ, Huiskes R (1990) Stereophotogrametry. In: Biomechanics of human movement (Berme N, Cappozzo A, eds), pp 108-127. Worthington, $\mathrm{OH}$ : Bertec Corporation.

Wood GA, Marshall RN (1986) The accuracy of DLT extrapolation in three-dimensional film analysis. J Biomech 19.9:781-785.

Young JZ (1965) The diameters of the fibers of the peripheral nerves of Octopus. Proc R Soc Lond [Biol] 162:47-79.

Young JZ (1971) The anatomy of the nervous system of Octopus vulgaris. Oxford: Clarendon. 